\title{
EVOLUÇÃO TEMPORAL DE INDICADORES DE SAÚDE BUCAL EM MUNICÍPIOS DO RIO GRANDE DO NORTE
}

\section{Temporal evolution of oral health indicators in municipalities of Rio Grande do Norte}

Isabela Dantas Torres de Araújo - Cirurgiã-dentista pela UFRN / Mestranda em Ciências Odontológicas com área de concentração em Clínicas Odontológicas pela UFRN / Especialista em Saúde Coletiva com Ênfase em Saúde da Família pela FACISA/UFRN / Especialista em Dentística pela Academia Norte Riograndense de Odontologia/UFRN. E-mail: isabeladta@gmail.com

Flávia Christiane de Azevedo Machado • Doutora em Saúde Coletiva pela Universidade Federal do Rio Grande do Norte - UFRN / Professora Adjunta I do Departamento de Saúde Coletiva da UFRN. E-mail: flavitamachado@yahoo.com.br

Autora responsável pela correspondência:

Isabela Dantas Torres de Araújo. E-mail: isabeladta@gmail.com 


\section{RESUMO}

Introdução: $O$ uso de indicadores é imprescindível para a vigilância à saúde na perspectiva de monitoramento e avaliação da qualidade dos serviços em prol da efetividade das políticas públicas. Objetivo: Descrever a evolução temporal dos indicadores de saúde bucal do Rol de Diretrizes, Objetivos, Metas e Indicadores 2013-2015 do município de Santa Cruz em comparação com Natal e o Rio Grande do Norte. Métodos: Estudo ecológico que utilizou dados disponíveis no DATASUS referentes aos anos de 2008 a 2015 dos indicadores de saúde bucal do Rol de Diretrizes, Objetivos, Metas e Indicadores 2013-2015. Resultados: Com relação ao indicador cobertura de equipes de saúde bucal, Santa Cruz ultrapassou os resultados do Estado do Rio Grande do Norte mostrando alta cobertura para a população. Já os indicadores média de escovação dental supervisionada e porcentagem de exodontias em relação aos procedimentos mostraram resultados insatisfatórios. Conclusão: Verifica-se que Santa Cruz possui um modelo de atenção odontológico de característica mutilatória e torna-se necessário a melhoria da organização dos serviços de saúde bucal a fim de possibilitar ao usuário do Sistema Único de Saúde uma atenção integral com práticas efetivas direcionadas à promoção da saúde e prevenção de doenças.

Palavras-chave: Saúde Pública. Saúde Bucal. Atenção Primária à Saúde. Indicadores Básicos de Saúde. Gestão da Informação em Saúde.

\section{ABSTRACT}

Introduction: The use of indicators is essential for health surveillance in the perspective of monitoring and evaluating the quality of services for the effectiveness of public policies. Objective To describe the temporal evolution of the oral health indicators of the Guidelines, Objectives, Targets and Indicators Role 2013-2015 in the municipality of Santa Cruz in the period 2008-2015 in comparison with Natal and Rio Grande do Norte. Methods: Ecological study of data available in DATASUS for the years 2008 to 2015 of oral health indicators of the Guidelines, Objectives, Targets and Indicators Role 2013-2015. Results: Regarding the indicator of oral health care coverage, Santa Cruz exceeded the results of the State of Rio Grande do Norte showing high coverage for the population. However, the indicators of average supervised dental brushing and percentage of dental extraction in relation to the procedures showed unsatisfactory results. Conclusion: It is verified that Santa Cruz has a dental care model of mutilatory characteristic and it is necessary to improve the organization of oral health services in order to allow Unified Health System's user an integral attention with effective practices directed to health promotion and disease prevention.

Keywords: Public Health. Oral Health. Primary Health Care. Health Status Indicators. Health Information Management. 


\section{Introdução}

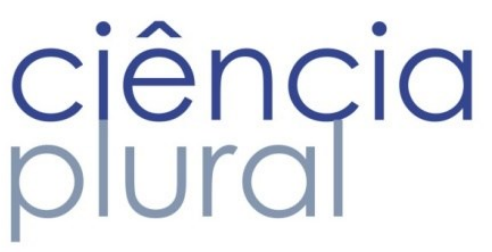

O atual modelo de atenção do Sistema Único de Saúde (SUS) é a vigilância à saúde, preconizando a integração entre ações de promoção à saúde, prevenção de doenças e recuperação da saúde. É uma ferramenta de transformação dos determinantes de saúde e doença por meio dos pilares intersetorialidade, território e participação popular. Ainda, é potencial para o monitoramento das condições de vida e saúde, reorientação dos serviços de saúde para vencer as desigualdades de cobertura, acesso e qualidade e controle social da informação em saúde 1 .

A utilização de indicadores sociais e de saúde é imprescindível para as práticas de vigilância à saúde uma vez que demonstram a situação de um indivíduo ou da população e servem como instrumento de monitoramento e avaliação da qualidade, desempenho e acesso que influencia na formulação de políticas públicas². Ademais, os indicadores são apresentados como taxas, proporções, médias e índices ao contrário das estatísticas públicas, como nos Censos Demográficos, que correspondem a um dado bruto não contextualizado por completo.

Neste sentido, é essencial instituir a prática da avaliação e monitoramento nos serviços de saúde e no âmbito da gestão em saúde. Não obstante, observa-se o interesse por parte de gestores e pesquisadores em institucionalizar a avaliação no SUS, todavia, processos avaliativos na esfera do SUS não representam atividades regulares na rotina dos serviços de saúde ${ }^{3-5}$.

O processo de avaliação em saúde constitui de procedimentos técnicos para produzir informações a respeito da situação de saúde, programas e projetos com a finalidade de garantir a eficácia, eficiência e efetividade, como também, aumentar a capacidade do gestor em mobilizar recursos e subsidiar a tomada de decisões ${ }^{5,6}$. Já o monitoramento em saúde constituiu-se em um método organizado e constante de fiscalização de uma política, programa ou projeto, fundamentado por informações que se traduzem em rápida avaliação situacional6.

Em relação ao modelo de atenção à saúde bucal, a Política Nacional de Saúde Bucal (PNSB/Brasil Sorridente) do Ministério da Saúde institucionalizada em 2004, reorientou-o de tal forma que o planejamento, a avaliação e monitoramento das ações, como também o uso de indicadores de saúde bucal, passaram a ser assimilados como instrumentos importantes para a sistematização da saúde bucal na Atenção Primária. Além disso, viabilizou a fluoretação das águas de abastecimento público, implantação dos Centros de Especialidades Odontológicas (CEO) e dos Laboratórios Regionais de Próteses Dentárias (LRPD)7,8. 


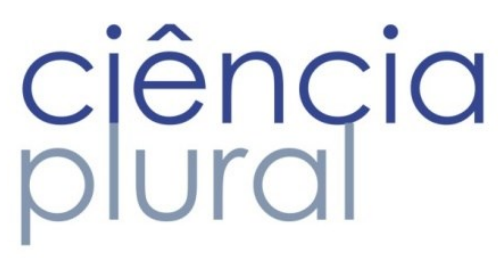

Em 2006 foi implementada a Portaria GM/MS nº 399, que divulga o Pacto pela Saúde - Consolidação do SUS e aprova as Diretrizes Operacionais nas suas três dimensões (Pacto pela Vida, Pacto de Gestão e Pacto em Defesa do SUS), sendo o Pacto, considerado uma ferramenta de regulação e orientação

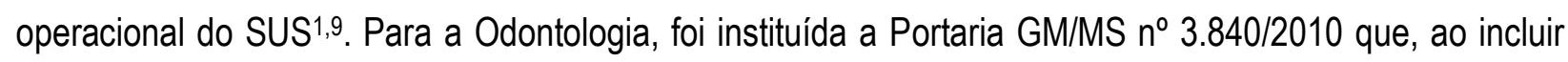
indicadores de saúde bucal, expressa relevância no processo de avaliação e monitoramento de programas e serviços da Atenção Primária que já havia sido visualizada na PNSB 10,11.

Nesse contexto de organização do SUS e como consequência do Decreto $n^{0} 7.508 / 2011$ que regulamenta a Lei Orgânica da Saúde, o Contrato Organizativo de Ação Pública da Saúde (COAP) é institucionalizado para também organizar de forma compartilhada as ações e os serviços na região de saúde, considerando as autonomias federativas, a fim de assegurar a integralidade da assistência à saúde e adequar o SUS com base no cidadão9.

Portanto a partir da consolidação do planejamento do SUS e a implementação do COAP, na $1^{\circ}$ Reunião Ordinária da Comissão Intergestores Tripartite (CIT) em 2013, foram estabelecidos princípios que orientaram a seleção das Diretrizes, Objetivos, Metas e Indicadores para o período 2013-2015. Nesse documento, no item III, o rol de indicadores para pactuação nacional, classificados em universais e específicos foram estabelecidos ${ }^{12}$.

Os indicadores universais retratam 0 acesso e a qualidade da organização em redes e são de pactuação comum e obrigatória em âmbito nacional. Já os indicadores específicos, representam as características epidemiológicas locais, de organização e desempenho do sistema sendo de pactuação obrigatória à medida que forem verificadas as particularidades no território ${ }^{12}$.

A partir dos atuais indicadores de saúde bucal pactuados por meio de metas nacionais como importantes ferramentas para a efetiva quantificação e avaliação das ações de saúde bucal no Brasil, objetivou-se descrever a evolução temporal desses indicadores no município de Santa Cruz/RN no período de 2008-2015 em comparação com Natal e o Rio Grande do Norte. Tais indicadores estão expostos no Caderno de Diretrizes, Objetivos, Metas e Indicadores 2013-2015 - Edição 2015 do Ministério da Saúde. Com isto, espera-se contribuir para o planejamento estratégico das ações de saúde bucal no município.

\section{MÉTODOS}

\section{Tipo de estudo}

Estudo ecológico, no qual foram analisados dados secundários disponiveis em base de dados contidos no Departamento de Atenção Básica (DAB), contemplado pelo DATASUS, referentes aos anos 


\section{ciência plural}

de 2008 a 2015 por meio dos indicadores de saúde bucal disponibilizados no Rol de Diretrizes, Objetivos, Metas e Indicadores 2013-2015. Esses indicadores são os que estão disponíveis para avaliar a perspectiva da atenção à saúde bucal, mas não são suficientes para caracterizar o modelo de atenção, uma vez que precisariam ser expandidos para uma melhor análise.

\section{Coleta de dados}

Os dados dos indicadores de saúde bucal foram obtidos em maio de 2017 e são de domínio público, provindos do Rol de Diretrizes, Objetivos, Metas e Indicadores 2013-2015, disponível no site do DATASUS. Para coletar os dados, basta acessar o link http://datasus.saude.gov.br/ e seguir o caminho: Acesso à Informação, TABNET, Indicadores de Saúde.

\section{Variáveis}

As variáveis coletadas no DATASUS estão identificadas no Quadro 1.

Quadro 1: Variáveis coletadas no DATASUS utilizadas para viabilizar a análise da evolução temporal de indicadores de saúde bucal no município de Santa Cruz/RN no período de 2008-2015.

\begin{tabular}{|c|c|c|c|}
\hline Variável & Descrição & Objetivo & Cálculo \\
\hline $\begin{array}{c}\text { Indicador } \\
4\end{array}$ & $\begin{array}{l}\text { Cobertura de } \\
\text { equipes de saúde } \\
\text { bucal. }\end{array}$ & $\begin{array}{l}\text { Medir a cobertura das equipes } \\
\text { de saúde bucal }\end{array}$ & $\begin{array}{l}\text { \{[(Soma da carga horária do } \\
\text { Cirurgião-Dentista / 40) } \times 3.000] / \\
\text { População no mesmo local e } \\
\text { período }\} \times 100\end{array}$ \\
\hline $\begin{array}{c}\text { Indicador } \\
5\end{array}$ & $\begin{array}{l}\text { Média de } \\
\text { escovação dental } \\
\text { supervisionada. }\end{array}$ & $\begin{array}{l}\text { Ponderar a proporção de } \\
\text { pessoas que tiveram acesso à } \\
\text { escovação dental com } \\
\text { orientação/supervisão de um } \\
\text { profissional de saúde bucal. }\end{array}$ & $\begin{array}{l}\text { [(n } \mathrm{n}^{0} \text { de pessoas participantes na } \\
\text { ação coletiva de escovação } \\
\text { dental supervisionada realizada } \\
\text { em determinado local em } 12 \\
\text { meses / 12) / População no } \\
\text { mesmo local e período] x } 100\end{array}$ \\
\hline $\begin{array}{c}\text { Indicador } \\
6\end{array}$ & $\begin{array}{lr}\text { Porcentagem } & \text { de } \\
\text { exodontias } & \text { em } \\
\text { relação } & \text { aos } \\
\text { procedimentos. }\end{array}$ & $\begin{array}{l}\text { Refletir, em percentual, a } \\
\text { proporção das exodontias de } \\
\text { dentes permanentes em } \\
\text { relação às demais ações } \\
\text { básicas individuais em } \\
\text { odontologia. Quanto menor o } \\
\text { percentual, maior a qualidade } \\
\text { do serviço odontológico do } \\
\text { município, visto que menos } \\
\text { dentes são extraídos e mais } \\
\text { procedimentos curativos são } \\
\text { realizados. }\end{array}$ & $\begin{array}{l}\left(\mathrm{n}^{0} \text { de exodontias de dentes }\right. \\
\text { permanentes em determinado } \\
\text { município e período / } \mathrm{n}^{0} \text { total de } \\
\text { ações básicas individuais em } \\
\text { odontologia de procedimentos } \\
\text { curativos selecionados no } \\
\text { mesmo local e período) } \times 100\end{array}$ \\
\hline
\end{tabular}




\section{ciência \\ plural}

\section{Análise de dados}

Os dados foram coletados e exportados para o programa Microsoft Office Excel 2016 para Windows onde foi construído o banco de dados.

Os dados referentes aos indicadores foram apresentados por meio de gráficos dos municípios de Santa Cruz, Natal e RN.

\section{Questões Éticas}

Os dados utilizados são de fonte secundária não envolvendo pesquisa com seres humanos.

\section{RESULTADOS}

Para efeito de comparação da caracterização dos municípios estudados, a Tabela 1 foi construída.

Tabela 1: Caracterização dos municípios estudados de acordo com a população, área territorial $\left(\mathrm{Km}^{2}\right)$ e densidade demográfica (Hab/Km²), segundo Censo Demográfico do IBGE, 2010.
Município
População
Área $\left(\mathrm{Km}^{2}\right)$
Densidade
Demográfica
$\left(\mathrm{Hab} / \mathrm{Km}^{2}\right)$

\begin{tabular}{llll}
\hline Natal & 803.739 & 171,15 & 4696,53 \\
Santa Cruz & 35.797 & 627,11 & 57,02 \\
RN & 3.168 .027 & $52.811,107$ & 59,99 \\
\hline
\end{tabular}

A Figura 1 mostra os percentuais de cobertura de equipes de saúde bucal (EqSB) nos municípios de Santa Cruz e Natal e do Estado do RN no período de 2008-2015.

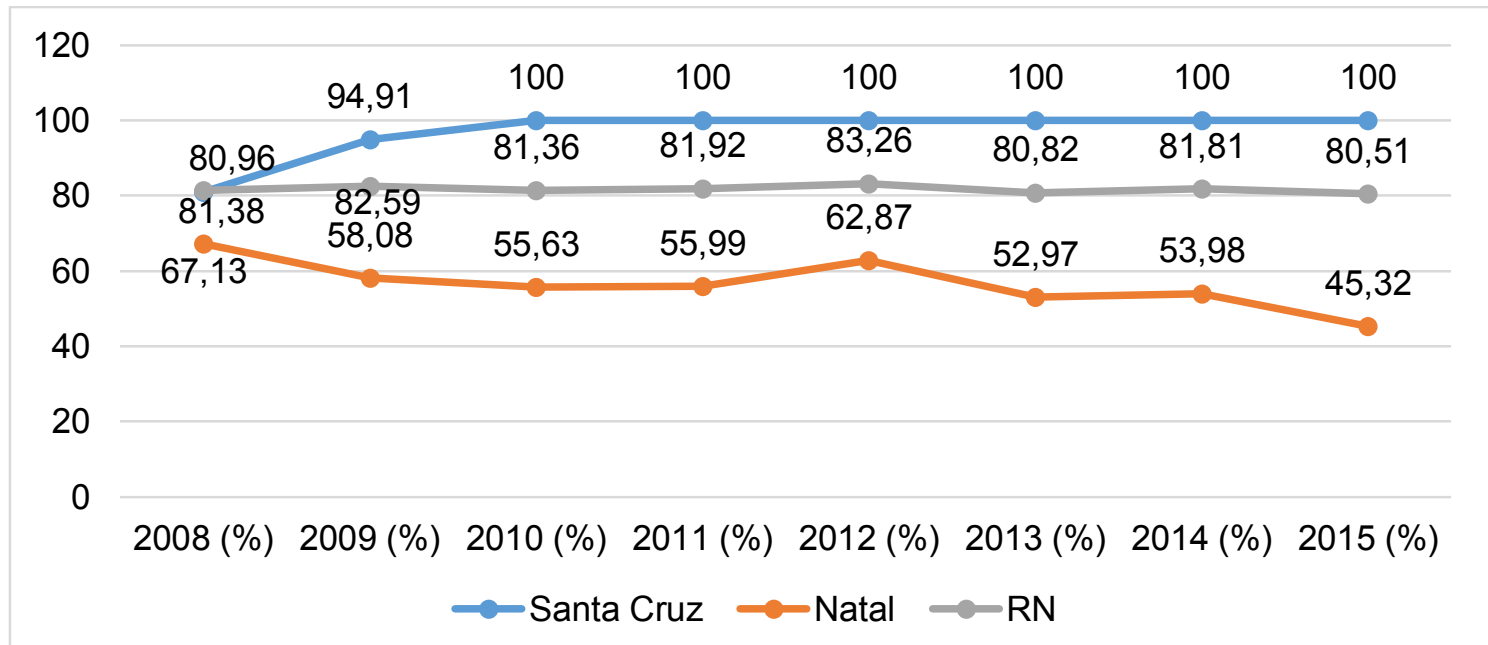

Figura 1: Resultados para o indicador 4, segundo os municípios de Santa Cruz e Natal e do Estado do RN, 2008-2015. Fonte: Brasil. Ministério da Saúde. DATASUS. 


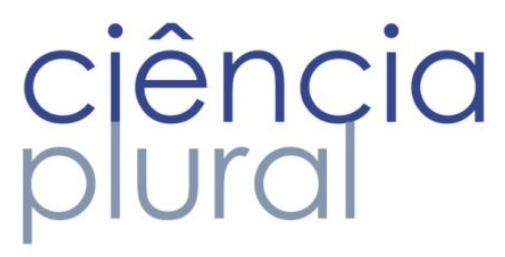

Para esse indicador, observa-se que o município de Natal apresenta os valores mais baixos em todos os anos representados enquanto que Santa Cruz, a partir do ano de 2010, apresentou $100 \%$ de cobertura das EqSB para a população.

A Figura 2 mostra os percentuais de média de escovação dental supervisionada nos municípios de Santa Cruz e Natal e do Estado do RN no período de 2008-2015.

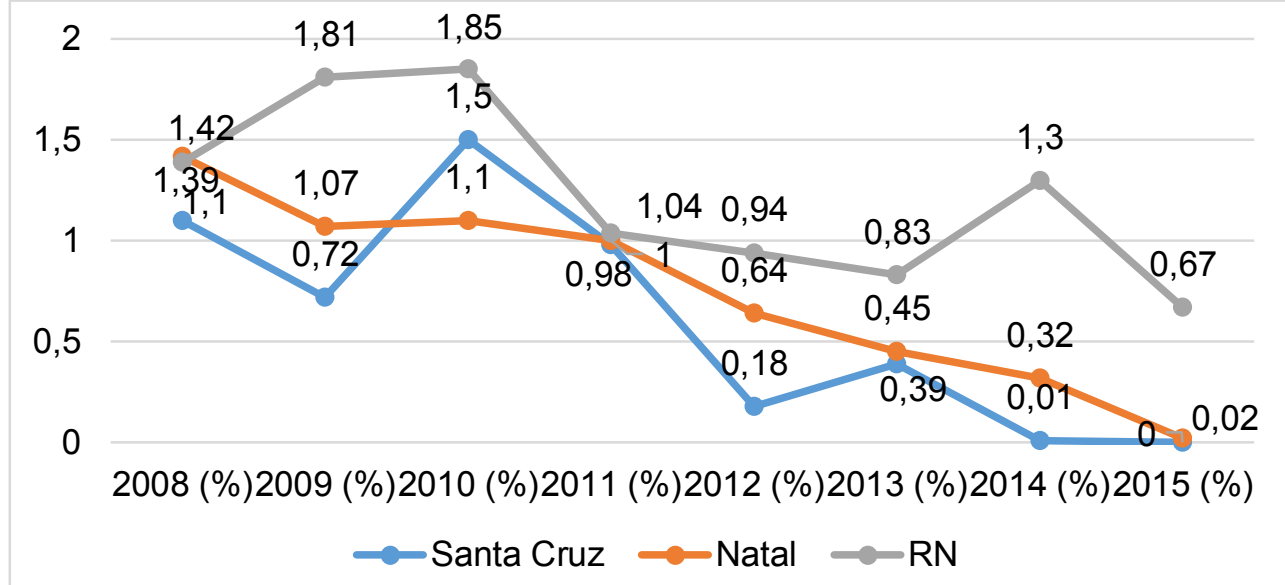

Figura 2: Resultados para o indicador 5, segundo os municípios de Santa Cruz e Natal e do Estado do RN, 2008-2015. Fonte: Brasil. Ministério da Saúde. DATASUS.

Com relação ao indicador de média de escovação dental supervisionada, observa-se que em 2008 a porcentagem desse indicador já se mostrava bem reduzida e ao longo dos anos a tendência foi diminuir ainda mais chegando em 2015 a 0\% para Santa Cruz e 0,02\% para Natal.

A Figura 3 mostra a proporção de exodontias em relação aos procedimentos odontológicos nos municípios de Santa Cruz e Natal e do Estado do RN no período de 2008-2015.

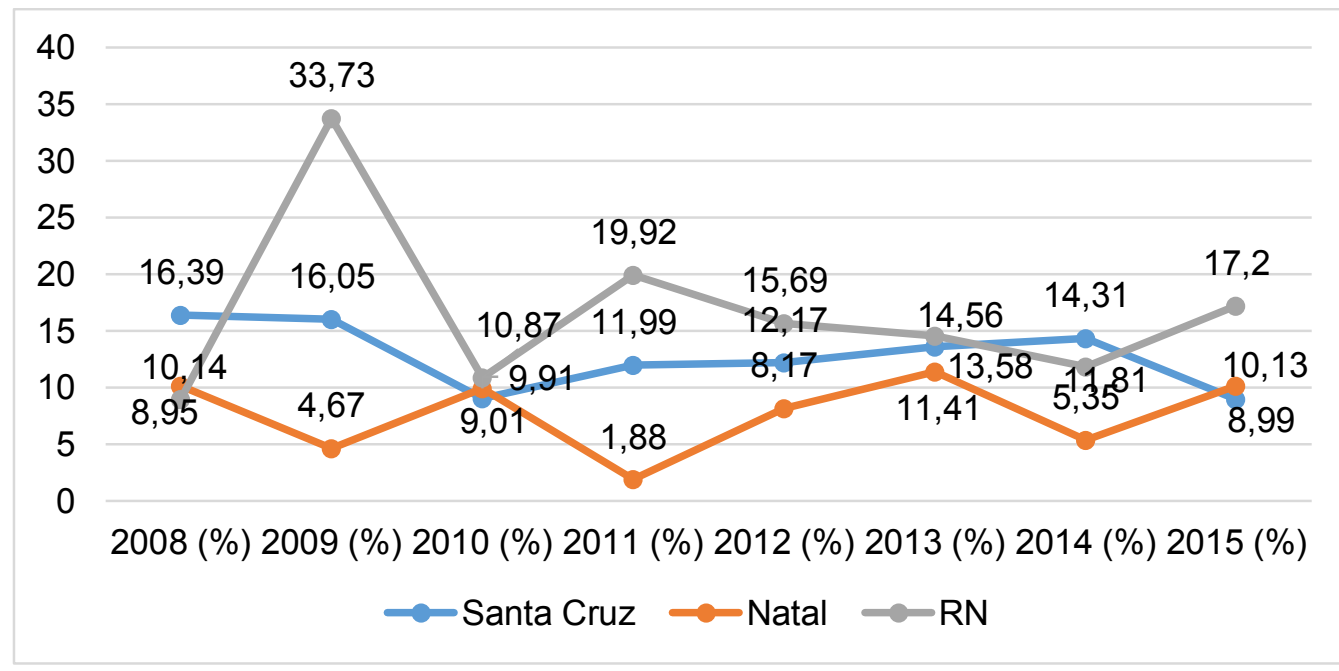

Figura 3: Resultados para o indicador 6, segundo os municípios de Santa Cruz e Natal e do Estado do RN, 2008-2015. Fonte: Brasil. Ministério da Saúde. DATASUS. 


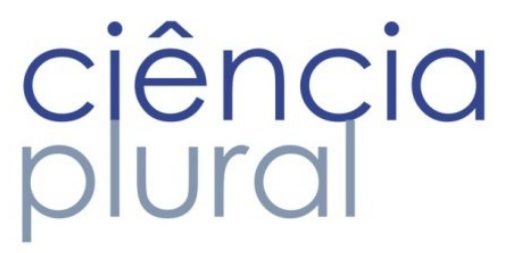

Para 0 indicador de proporção de exodontias em relação aos procedimentos odontológicos, verifica-se uma queda em Santa Cruz a partir do ano de 2010 (9,01\%) com sucessivo aumento em 2011 e novamente em 2015 há uma queda expressiva para 8,99\%. No município do Natal houve uma oscilação ainda mais significativa desse indicador entre os anos representados neste estudo.

\section{DISCUSSÃO}

Para que seja alcançado, na atenção primária, um efetivo planejamento em saúde bucal, é necessário retomar a importância da epidemiologia com destaque para a relevância dos sistemas de informação em saúde (SIS) e seus indicadores. A construção e utilização desses indicadores é fundamental nas condutas de avaliação e controle da situação de saúde de populações expondo as mudanças nestes perfis 9,13.

A avaliação dos indicadores de saúde bucal pactuados torna-se fundamental uma vez que retrata em números a situação em que se encontra a saúde bucal na atenção primária. É importante ressaltar que a interpretação dos indicadores é necessária para a elaboração das ações e serviços de saúde bucal nos municípios, mesmo que possam ocorrer falhas na obtenção e análise desses indicadores. Os indicadores apresentam um panorama muito aproximado das realidades locais, sendo primordial para conduzir futuras ações ${ }^{9}$.

Nesse contexto, de acordo com o Caderno de Diretrizes, Objetivos, Metas e Indicadores 2013-2015, novamente afirma-se a importância dos indicadores para as fases de monitoramento e avaliação do serviço de saúde uma vez que possibilitam observar o cumprimento das metas pactuadas. Ainda, é possível analisar criticamente os resultados obtidos e dessa forma, contribuir na tomada de decisão para melhorar continuamente os processos organizacionais e o desempenho do serviço ${ }^{12}$.

Para começar a discussão dos resultados encontrados, o indicador 4 - cobertura de EqSB - mostra o acesso da população aos serviços de saúde bucal, portanto sua interpretação afirma que quanto maior a cobertura, maior será a oferta e 0 acesso aos serviços odontológicos.

Foi verificado que houve uma expansão significativa da cobertura da ESF entre 1998 e 2004 influenciada pela criação da PNSB, o que beneficiou consideravelmente os municípios de menor Índice de Desenvolvimento Humano (IDH) e que no ano de 2004, a proporção dessa cobertura na Região Nordeste já atingia $55 \%$ em contraste com a Região Sul (38\%) sucedendo de forma continuada ${ }^{14}$.

Um estudo que avaliou a incorporação da saúde bucal no Programa Saúde da Família (PSF) no RN afirmou que grande parte dos municípios não obteve avanços no modelo assistencial em saúde bucal após sua implementação no PSF e do universo de 19 municípios, apenas 9 foram categorizados como 


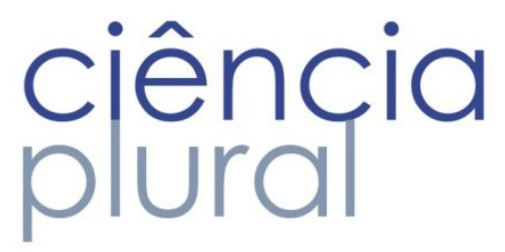

insatisfatórios com relação às mudanças apresentadas e aqueles que mais avançaram foram os que apresentaram melhores condições de vida ${ }^{15}$.

Foram observados também os itens mais críticos nos municípios, destacando-se que, até o momento do estudo, não foram encontradas melhorias nas relações de trabalho, referenciamento de pacientes, elaboração de ações intersetoriais, nas estratégias de programação, na infraestrutura e presença de protocolos de atendimento e relatórios de ações desenvolvidas ${ }^{15}$.

Então em 2014, verifica-se a meta de cobertura populacional para o Rio Grande do Norte de $80 \%{ }^{16} \mathrm{e}$ de acordo com os dados da pesquisa no SIS, o Estado atingiu $81,81 \%$ e os municípios de Santa Cruz e Natal atingiram $100 \%$ e 53,98\% respectivamente. Assim sendo, Santa Cruz superou a meta e o resultado do RN em 2014 e 2015, no entanto Natal obteve resultados muito abaixo do esperado.

De acordo com os dados mais recentes do DATASUS, o município do Natal possui 55 unidades de saúde sendo que, por meio do site da prefeitura, verifica-se que pelo menos $38(69 \%)$ dessas unidades se enquadram no modelo de ESF em contraste com Santa Cruz, que possui apenas 8 unidades de saúde e todas seguem o modelo de ESF.

Isto seria um dos fatores para explicar a baixa cobertura das equipes de saúde bucal. Todavia, pelo motivo acima colocado não implicaria uma deficiência de acesso aos serviços de saúde bucal, uma vez que as Unidades Básicas de saúde tradicionais disponibilizam a assistência odontológica.

A insuficiente cobertura populacional em Natal pode se correlacionar com a política de financiamento para a inserção de equipes, uma vez que há maior incentivo aos municípios de pequeno porte priorizando a interiorização da ESF e a pressão praticada pelo setor privado.

A estratégia traz consigo um conceito ampliado de saúde ${ }^{17}$, visa potencializar a reorientação do processo de trabalho em saúde e, por conseguinte, desfazer a fragmentação do cuidado ${ }^{1}$. Ademais, permite que as unidades de saúde sejam mais acolhedoras, com atendimentos de maior qualidade e resolubilidade e o aumento de sua cobertura proporciona significativa equidade 18,19.

Nota-se a importância da implantação e expansão do modelo da ESF 8,19 associado à inserção da integralidade do cuidado mesmo que haja dificuldades na modificação dos processos de trabalho vigentes justificados pela persistência do modelo biomédico cujo foco se dá às doenças agudas. Ademais, verificam-se avanços no trabalho em saúde bucal relativos às ações educativas, de educação permanente, acolhimento, vínculo e responsabilização ${ }^{20}$.

A integralidade, ampliação e qualificação da assistência e o acesso, são assuntos comuns na literatura e podem auxiliar no aprimoramento e direcionamento de políticas públicas, superação de 


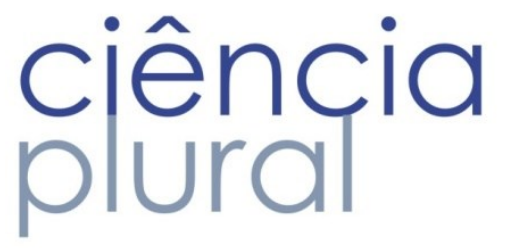

desigualdades no acesso, obtenção da equidade e de uma integralidade mais efetiva relacionada às práticas e ao trabalho em equipe ${ }^{19,20}$.

Acerca do indicador 5, média de escovação dental supervisionada, faz referência ao acesso à orientação para prevenção de afecções orais, principalmente no que tange à cárie e doença periodontal. Para o estado do RN, a meta em 2014 foi de 3\%, no entanto verifica-se a tendência de diminuição da porcentagem desse indicador tanto para os municípios em estudo quanto para o próprio estado, cujos índices foram de 0,01 (Santa Cruz), 0,32 (Natal) e 1,30 (RN).

Embora a população tenha maior acesso aos dentifrícios fluoretado, levando à uma proteção contra a cárie e a doença periodontal, é importante que as ações de escovação supervisionada prevaleçam para que, de certa forma, seja assegurada o futuro declínio da cárie ${ }^{21}$, redução da necessidade de procedimentos restauradores, exodontias e, por conseguinte, redução dos gastos públicos. No entanto, é importante lembrar que ainda existem municípios no Brasil que não há fluoretação das águas de abastecimento 8 .

Ademais, é importante colocar que o auxiliar de saúde bucal (ASB) e o técnico de saúde bucal (TSB) possuem autonomia para realizar a escovação dental supervisionada e, portanto, a ausência desses profissionais na equipe de saúde bucal prejudica os indicadores de produtividade e efetividade.

O intuito da implementação desse tipo de ação é motivar o paciente a desenvolver práticas que o faça manter uma condição bucal satisfatória. Ainda, a educação da população é um dos caminhos a seguir para o controle e prevenção de doenças ${ }^{8,17}$ uma vez que é verificada a efetividade dessas ações na melhoria das condições bucais e mudança de hábitos de saúde ${ }^{22}$.

Isto poderia estar relacionado à queda do indicador, sendo possível que as EqSB estejam realizando ações diversas de educação em saúde, além de escovação supervisionada. Desta forma, essa queda, se de fato acompanhada pelo aumento de atividades de educação em saúde diversas, não implica em um resultado insatisfatório.

Com relação ao indicador 6, proporção de exodontias em relação aos procedimentos odontológicos, é traduzido em quanto menor esse percentual, menos mutilatório é o serviço em questão e, destarte, verifica-se um serviço de caráter preventivo e conservador. A meta pactuada para o RN com relação a esse indicador foi de $5 \%$ para o ano de 2014. Nota-se neste ano que apenas Natal se aproximou da meta $(5,35 \%)$ enquanto que Santa Cruz obteve $14,31 \%$ e o RN, $11,81 \%$.

Ademais, ao analisar a Figura 3 , é possível verificar a tendência de alto índice de exodontias ao longo dos anos de 2008 a 2015 e, portanto, ainda é predominante um serviço odontológico de caráter mutilatório 


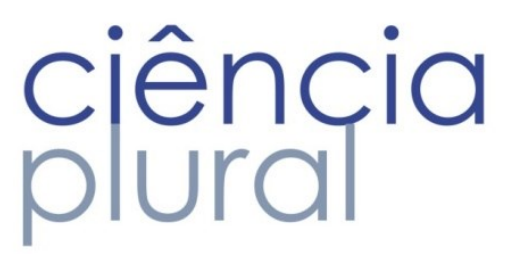

muitas vezes associado às condições socioeconômicas da população, escolaridade, falta de acesso e pela cultura de substituição dos dentes por próteses ${ }^{23}$.

É importante lembrar também, que Natal possui menor cobertura pela ESF e menor porcentagem de exodontias comparado com Santa Cruz que possui dados opostos, ou seja, Natal apresenta uma assistência odontológica com característica mais curativa e isso pode ser explicado pelo modelo de atenção de cada município (mutilatório ou curativo), pelas características da população (mais informada ou não) como também pela subnotificação dos dados que, infelizmente, se trata de um problema comum dos SIS 24 .

Outrossim, extrações dentárias possuem forte associação com a reabilitação protética e, com a implantação do Brasil Sorridente, essa reabilitação se tornou uma das metas da PNSB com o objetivo de ampliar a quantidade de laboratórios e a oferta de próteses. Esse fato também pode aumentar o número de exodontias uma vez que se torna mais prático e algumas vezes menos oneroso para o indivíduo substituir um elemento dentário que frequentemente necessitava de tratamento.

\section{CONCLUSÕES}

Os sistemas de informação em saúde são instrumentos essenciais de gestão para a avaliação, monitoramento, organização, funcionamento, planejamento das ações de saúde e tomada de decisão. Além disso, podem auxiliar os gestores na reorientação ou manutenção das políticas públicas vigentes. No entanto, para a atenção à saúde bucal, existem poucos indicadores e são caracterizados como indicadores de assistência.

Verifica-se neste estudo que o município de Santa Cruz possui características de um modelo de atenção odontológico mutilador em comparação com o município do Natal uma vez que apresentou resultados insatisfatórios para os indicadores 5 e 6 levando-se também em consideração às metas pactuadas para o estado do RN.

Ainda, Santa Cruz ultrapassou os resultados do Estado do RN no que tange ao indicador 4, o que mostra alta cobertura de EqSB para essa população.Destaca-se a necessidade de aumentar a cobertura populacional do município do Natal para construir uma rede de cuidados mais ampla que envolva todos os níveis de atenção.

O estudo apresenta limitações características do próprio desenho uma vez que estudos ecológicos possuem baixo poder analítico e pouco desenvolvimento das técnicas de análise de dados. Ademais, 


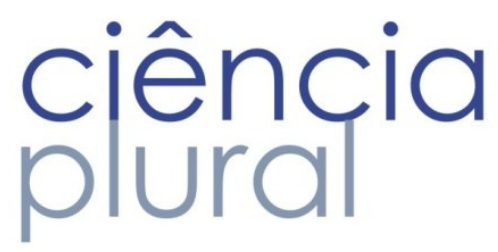

como se trata de dados secundários, deve-se considerar a possibilidade da fragilidade e do controle de qualidade dos dados coletados nos SIS. Com base neste perfil situacional do município de Santa Cruz, é substancial a melhoria da organização dos serviços de saúde bucal com o objetivo de possibilitar ao usuário do SUS uma atenção integral com práticas efetivas voltadas à promoção da saúde e prevenção de doenças.

\section{Referências}

1. Oliveira CM, Cruz MM. Sistema de Vigilância em Saúde no Brasil: avanços e desafios. Saúde em Debate. 2015;39(104):255-67.

2. Avelino CCV, Goyatá SLT, Nogueira DA, Rodrigues LBB, Siqueira SMS. Qualidade da atenção primária à saúde: uma análise segundo as internações evitáveis em um município de Minas Gerais, Brasil. Cien Saude Colet. 2015;20(4):1285-93. Disponível em: http://www.scielosp.org/pdf/csc/v20n4/pt_1413-8123-csc-20-04-01285.pdf

3. Carvalho ALB, Souza MF, Shimizu HE, Senra IMVB, Oliveira KC. A gestão do SUS e as práticas de monitoramento e avaliação: possibilidades e desafios para a construção de uma agenda estratégica.

Cien Saude Colet. 2012;17(4):901-11. Disponivel em: http://repositorio.unb.br/bitstream/10482/12732/1/ARTIGO_GestaoSUSPraticas.pdf

4. Furtado JP, Vieira-da-Silva LM. A avaliação de programas e serviços de saúde no Brasil enquanto espaço de saberes e práticas. Cad Saude Publica. 2014;30(12):2643-55. Disponivel em: http://www.scielo.br/scielo.php?script=sci_artext\&pid=S0102-311X2014001202643\&lng=pt\&tlng=pt

5. Pereira BS, Tomasi E. Instrumento de apoio à gestão regional de saúde para monitoramento de indicadores de saúde. Epidemiol Serv Saude. 2016;25(2):411-8. Disponível em: http://www.scielosp.org/pdf/ress/v25n2/2237-9622-ress-25-02-00411.pdf

6. Jannuzzi PM. Avaliação de programas sociais: conceitos e referenciais de quem a realiza. Estud em Avaliação Educ. 2014;25(58):22-42. Disponivel em: http://publicacoes.fcc.org.br/ojs/index.php/eae/article/view/2916/2768

7. Brasil. Ministério da Saúde. Secretaria de Atenção à Saúde. Departamento de Atenção Básica. Coordenação Nacional de Saúde Bucal. Diretrizes da Política Nacional de Saúde Bucal. Brasília: Ministério da Saúde, 2004. p. 16. Disponível em: http://bvsms.saude.gov.br/bvs/publicacoes/politica_nacional_brasil_sorridente.pdf 


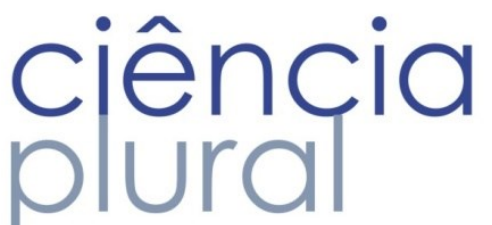

8. Scarparo A, Zermiani TC, Ditterich RG, Pinto MHB. Impacto da Política Nacional de Saúde Bucal Programa Brasil Sorridente - sobre a provisão de serviços odontológicos no Estado do Rio de Janeiro. Cad Saúde Coletiva. 2015;23(4):409-15. Disponível em:

http://www.scielo.br/scielo.php?script=sci_arttext\&pid=\$1414462X2015000400409\&lng=pt\&nrm=iso \&tlng=en

9. Fernandes JKB, Pinho JRO, Queiroz RCS, Thomaz EBAF. Avaliação dos indicadores de saúde bucal no Brasil: tendência evolutiva pró-equidade? Cad saúde pública. 2016;32(2):1-18. Disponível em: http://www.scielo.br/scielo.php?script=sci_arttext\&pid=S0102-

311X2016000200701\&lng=en\&nrm=iso\&tlng=pt

10. Brasil. Ministério da Saúde. Portaria no 399/GM, de 22 de fevereiro de 2006. Divulga o Pacto pela Saúde 2006 - Consolidação do SUS e aprova as Diretrizes Operacionais do Referido Pacto. Diário Oficial da União, Brasília, DF, 22 fev. 2006. Disponível em: http://bvsms.saude.gov.br/bvs/saudelegis/gm/2006/prt0399_22_02_2006.html

11. Brasil. Ministério da Saúde. Portaria no 3.840, de 7 de dezembro de 2010. Inclui a Saúde Bucal no Monitoramento e a Avaliação do Pacto pela Saúde, e estabelece as diretrizes, orientações e prazos do processo de ajuste de metas para o ano de 2011. Brasília; 2010.

12. Brasil. Ministério da Saúde. Secretaria de Gestão Estratégica e Participativa. Departamento de Articulação Interfederativa. Caderno de Diretrizes, Objetivos, Metas e Indicadores : 2013-2015. Brasília; 2013. Disponível em:

http://bvsms.saude.gov.br/bvs/publicacoes/caderno_diretrizes_objetivos_2013_2015_2edicao.pdf

13. Marques AB, Oneda G, Buffon MCM, Ditterich RG. Sistemas de Informação como ferramenta de monitoramento das ações de saúde bucal na Estratégia Saúde da Família da região metropolitana de Curitiba-PR. Rev Bras Pesq Saúde. 2014;16(1):82-9. Disponível em: http://www.periodicos.ufes.br/RBPS/article/viewFile/8494/5990

14. Conill EM. Ensaio histórico-conceitual sobre a Atenção Primária à Saúde: desafi os para a organização de serviços básicos e da Estratégia Saúde da Família em centros urbanos no Brasil. Cad Saude Publica. 2008;24(1):7-27. Disponível em: http://www.scielo.br/pdf/csp/v24s1/02.pdf

15. Souza TMS, Roncalli AG. Saúde bucal no Programa Saúde da Família: uma avaliação do modelo assistencial. Cad Saude Publica. 2007;23(11):2727-39.

16. Brasil. Prefeitura Municipal do Natal. Secretaria Municipal de Saúde. Relatório anual de gestão (RAG). 2014. p. 130. Disponível em: https://natal.rn.gov.br/sms/paginas/File/relatorios-degestao/relatorio_de_gestao_sms_2014.pdf

17. Thurow LL, Castilhos ED, Costa JSD. Comparação das práticas odontológicas segundo modelos de atendimento: tradicional e da Saúde da Família, Pelotas-RS, 2012-2013. Epidemiol Serv Saúde. 
2015;24(3):545-50. Disponível em: http://www.scielo.br/pdf/ress/v24n3/2237-9622-ress-24-0300545.pdf

18. Coutinho AT, Popim RC, Carregã K, Spiri WC. Integralidade do cuidado com o idoso na estratégia de saúde da família: visão da equipe. Esc Anna Nery. 2013;17(4):628-37. Disponível em: http://www.gnresearch.org/doi/10.5935/1414-8145.20130005

19. Malta DC, Santos MAS, Stopa SR, Vieira JEB, Melo EA, Reis AAC. A Cobertura da Estratégia de Saúde da Família (ESF) no Brasil, segundo a Pesquisa Nacional de Saúde, 2013. Cien Saude Colet. 2016;21(2):327-38. Disponivel em: http://www.scielo.br/scielo.php?script=sci_arttext\&pid=S1413$81232016000200327 \&$ lng=pt\&tlng=pt

20. Scherer $\mathrm{Cl}$, Scherer MDA. Avanços e desafios da saúde bucal após uma década de Programa Brasil Sorridente. Rev Saude Publica. 2015;49.

21. Frazão P. Custo-efetividade da escovação dental supervisionada convencional e modificada na prevenção da cárie em molares permanentes de crianças de 5 anos de idade. Cad Saude Publica. 2012;28(2):281-90.

22. Silva GG, Carcereri DL, Amante CJ. Estudo qualitativo sobre um programa de educação em saúde bucal. Cad Saúde Coletiva. 2017;25(1):7-13. Disponivel em: http://www.scielo.br/scielo.php?script=sci_arttext\&pid=S1414-462X2017000100007\&lng=pt\&tlng=pt

23. Pimentel BV, Zermiani TC, Ditterich RG, Pecharki GD. A utilização dos indicadores de saúde bucal e desenvolvimento humano no monitoramento da atenção básica nos municípios da região metropolitana de Curitiba - PR. Vol. 15, Revista Espaços para a Saúde. Londrina; 2014. 42-52 p. Disponivel em: http://www.uel.br/revistas/uel/index.php/espacoparasaude/article/view/18250/pdf_39

24. Siqueira CL, Carvalho e Silva C, Teles JKN, Feldman LB. Gerenciamento de risco: percepção de enfermeiros em dois hospitais do Sul de Minas Gerais, Brasil. Rev Min Enferm. 2015;19(4):919-26. Disponivel em: http://www.revenf.bvs.br/pdf/reme/v19n4/v19n4a10.pdf

Submetido: 06/07/2018

Aprovado: 02/01/2019 